УДК $620.19,621.039 .586,621.039 .524 .2 .034 .3$

\title{
ИССЛЕДОВАНИЯ ВЫСОКОТЕМПЕРАТУРНОЙ КОРРОЗИИ SIС-ПОКРЫТИЯ НА ГРАФИТЕ
}

\section{Аскербеков Саулет Каныбекулы',} askerbekov@physics.kz

\author{
Чихрай Евгений Васильевич', \\ chikhray@physics.kz
Понкратов Юрий Валентинович², ponkratov@nnc.kz

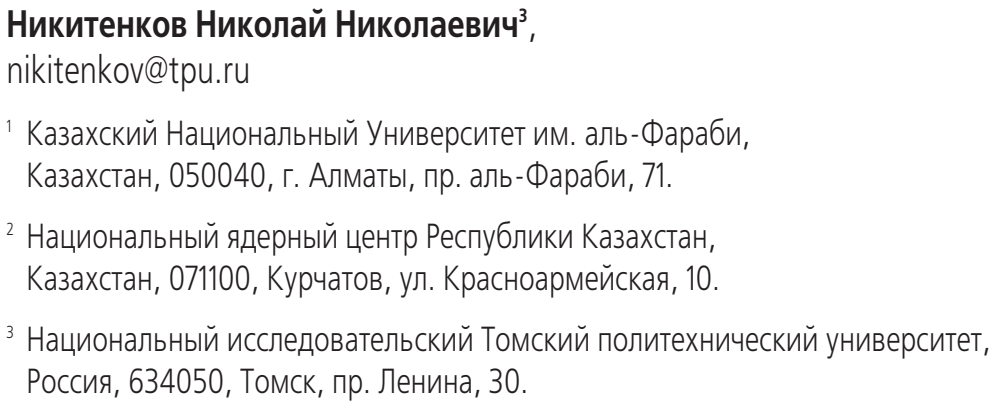

Актуальность исследования обусловлена перспективой создания казахстанского высокотемпературного газоохлаждаемого реактора, в котором будет применен новый набор реакторных графитов и пиролитических углеродных покрытий элементов топлива. На топливных элементах реактора будут использованы покрытия из карбида кремния (SiC) поверх графитой или пироуглеродной основы.

Цель: определить скорость и особенности протекания высокотемпературной коррозии реакторного графита с SiC-покрытием в диапазоне температур от 750 до 1400 ㄷ при начальном давлении паров воды в камере 100 Па.

Объект: изотропный мелкозернистый графит марки IG-110 с защитным SiC-покрытием толщиной 200 мк.

Методы: десорбция продуктов коррозии с поверхности и объема образца в присутствии паров воды при различных исследуемых температурах, масс-спектрометрическая регистрация состава газов в рабочей камере, микроструктурные исследования, Рамановская спектроскопия, энерго-дисперсная рентгеновская спектроскопия.

Результаты. Установлено, что реакция SiC-покрытия с водяным паром при давлении 100 Па и выше происходит с образованием защитного слоя аморфного диоксида кремния $\mathrm{SiO}_{2}$ (пассивное окисление SiC). Коррозия SiC в парах воды при температурах

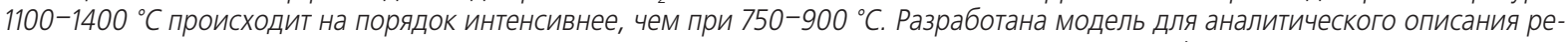
зультатов экспериментов и рассчитаны значения скорости реакции водяного пара с покрытием SiC при температурах 1400, 1300, 1200, 1100 и $1000^{\circ} \mathrm{C}$. Анализ изменения микроструктуры образцов после коррозионного эксперимента свидетельствует об образовании на поверхности карбида кремния защитного слоя $\mathrm{SiO}_{2}$, характерного для механизма пассивного окисления SiC. Результаты исследования позволяют заключить, что реакторный графит с SiC-покрытием обладает лучшими антикоррозионными свойствами по сравнению с чистым графитом, а SiC может быть применен для химической защиты графитовых и углеродных слоев топлива высокотемпературного газоохлаждаемого реактора.

\section{Ключевые слова:}

Реакторный графит, SiC-покрытие, ВТГР, коррозия, водяной пар.

\section{Введение}

Тематика материаловедческих исследований в области ядерных и термоядерных установок является традиционной для казахстанской науки [1]. На установках Института Атомной Энергии (ИАЭ), Института Ядерной Физики (ИЯФ) и Института экспериментальной и теоретической физики было исследовано множество конструкционных и функциональных материалов для будущих ядерных и термоядерных реакторов [2-11].

Одним из таких исследований являются эксперименты по коррозии SiC-покрытий реакторного графита, результаты которых представлены в настоящей статье.

Условия использования графита и пиролитического углерода в элементах топлива и конструкции активной зоны реакторов деления (высокие температуры и давления) подразумевают постоянное химическое воздействие на них имеющихся в охладителе остаточных примесей кислорода и воды (водяного пара). Это особенно актуально для реакторов типа ВТГР (высокотемпературных газоохлаждаемых реакторов).

Изучение возможных направлений изменения технологических параметров реакторного графита ВТГР при его контакте с воздухом и водой в ходе штатной эксплуатации и при проектных авариях (вплоть до разрушения его структуры) является актуальной и обязательной задачей исследований, обеспечивающей безопасность этого типа реакторов.

Для снижения возможного разрушения графитовых материалов газового реактора предлагается 


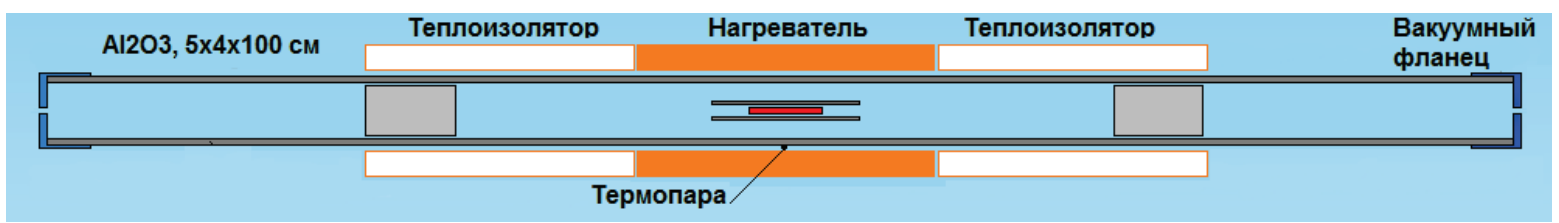

Puc. 1. Схема расположения элементов рабочей камеры установки для исследования коррозии графитов на базе трубчатой печи GSL-1600

Fig. 1. Layout of the working chamber elements of the facility for studying graphite corrosion on the basis of GSL-1600 tube furnace

использовать разнообразные покрытия [12-14], в частности покрытия на основе карбида кремния [15].

Таким образом, проведение экспериментов по исследованию взаимодействия карбид-кремниевых защитных покрытий с химическими активными газами (воздух, вода и т. д.) остается одной из актуальных и приоритетных задач [15-26]. Задачей настоящего исследования было оценить скорость и закономерности протекания высокотемпературной коррозии реакторного графита с $\mathrm{SiC}-$ покрытием в диапазоне температур 750-1400 ${ }^{\circ} \mathrm{C}$ в присутствии паров воды ( 100 Па).

\section{Исследуемый материал}

В работе проведены испытания нового защитного покрытия $\mathrm{SiC}$ на изотропном мелкозернистом графите марки IG-110 и исследована его защитная способность. SiC-покрытие толщиной 200 мк наносилось на графит методом пиролиза метилтрихлорсилана $\mathrm{CH}_{3} \mathrm{SiCl}_{3}$ [27]. По результатам рентгенофазового анализа структура полученного покрытия соответствует $\beta$-фазе карбида кремния (3C-SiC) с кубической решеткой пространственной группы F43m (216) [19]. Полученный образец графита имел форму параллелепипеда размерами $4 \times 4 \times 45$ мм. Образец вырезан из массивного блока графита перпендикулярно оси его технологическо- го сжатия при изготовлении. При этом образец весил примерно один грамм, плотность графита была

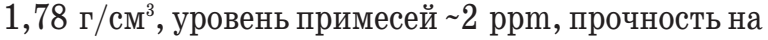
изгиб 39 МПа, твердость по Шору 51.

\section{Экспериментальная установка}

Коррозионные эксперименты с образцами реакторного графита проводились на установке CorrSiC'a [19]. Основой установки является горизонтальная вакуумная печь типа GSL-1600 (рис. 1) с двумя боковыми загрузочными фланцами. Вакуумная камера печи образуется основной коррундовой трубой $\left(\mathrm{Al}_{2} \mathrm{O}_{3} \sim 99,5 \%\right)$ и обеспечивает программируемый нагрев исследуемого образца в газовой среде или в вакууме до $1600{ }^{\circ} \mathrm{C}$. Толщина стенки трубы 5 мм, внутренний диаметр 40 мм, длина зоны нагрева 400 мм. Вакуумное уплотнение вынесенных концов трубы камеры на каждом из торцов создается двумя прижимными кольцами высококачественного термостойкого силикона, обеспечивающими натекание воздуха не более 0,0025 Па•л/мин. Печь обеспечивает 150 мм зоны однородного нагрева при точности регулировки температуры $\pm 1{ }^{\circ} \mathrm{C}$. Температура измеряется защищенной штатной термопарой (тип $\mathrm{S}, 87 \%$ $\mathrm{Pt} / 13 \% \mathrm{Rh}-\mathrm{Pt}$, максимальная погрешность $\left.\pm 1,5{ }^{\circ} \mathrm{C}\right)$, расположенной, как показано на рис. 1. Одновременно в печь можно загрузить до восьми

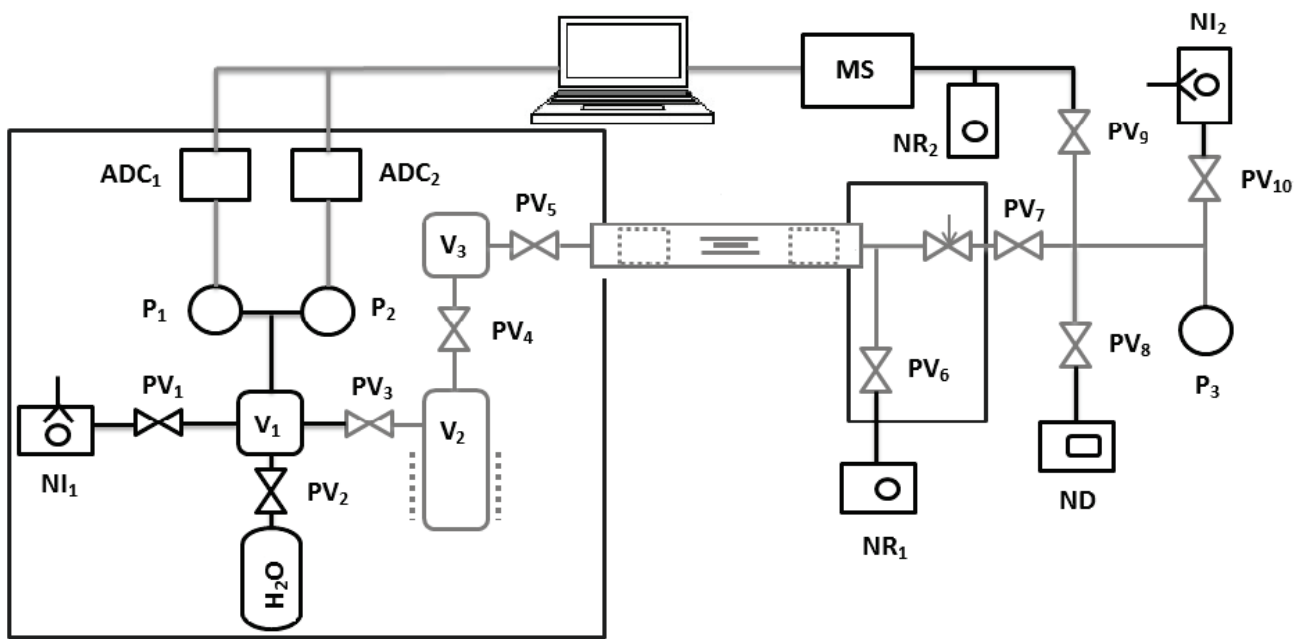

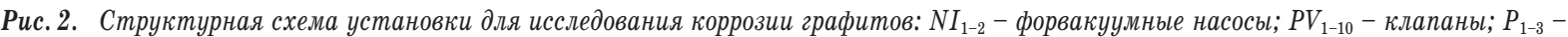
датчики давления; $V_{1-3}$ - вакуумные камеры; $N R_{1-2}-$ турбололекулярные насосы; $N D$ - магниторазрядный насос; $M S$ - массспектрометр; $A D C_{1-2}$ - аналого-иифровые преобразователи

Fig. 2. Block diagram of the facility for studying graphite corrosion: $N I_{1-2}-$ forevacuum pump; $P V_{1-10}-$ valve; $P_{1-3}-$ pressure sensor; $V_{1-3}-$ vacuum chamber; $N R_{1-2}$ - turbomolecular pump; $N D$ - magnetic discharge pump; $M S$ - mass spectrometer; $A D C_{1-2}-$ analog-to-digital converter 
образцов указанного выше размера. Защита боковых загрузочных фланцев и силиконовых уплотнительных колец от перегрева излучением осуществляется путем установки в камеру печи специальных тепловых экранов, изготовленных из корунда. Тепловые экраны образуют зазор (3-5 мм) со стенками печи, что удерживает температуру фланцев ниже $150{ }^{\circ} \mathrm{C}$ даже при температуре в центре $1500{ }^{\circ} \mathrm{C}$, не нарушая при этом движения медленных газовых потоков.

Для мониторинга состава газовой среды в рабочей камере установки используется квадрупольный масс-анализатор SRS CIS-100 (Stanford Research Systems, Closed Ion Source Gas Analyzer, США). Дополнительно установка оснащена передвижным постом высоковакуумной безмасляной откачки TPS-Mobile (Agilent Technologies), что позволяет эффективно откачивать рабочую камеру и исключить возможность попадания высокомолекулярных органических соединений в вакуумную и аналитическую системы установки. Структурная схема установки приведена на рис. 2 , а ее технические параметры - в табл. 1. На рис. 3 показан внешний вид установки.

таблица 1. Основные параметры установки для исследования коррозии графита

Table 1. Main parameters of the facility for studying graphite corrosion

\begin{tabular}{|l|c|c|}
\hline Teмпературный диапазон, ${ }^{\circ} \mathrm{C}$ & мин./min & 25 \\
\cline { 2 - 3 } Temperature range, ${ }^{\circ} \mathrm{C}$ & макс./max & 1600 \\
\hline Скорость нагрева/охлаждения, ${ }^{\circ} \mathrm{C} /$ мин & мин./min & 0 \\
\cline { 2 - 3 } Speed of heating/cooling, ${ }^{\circ} \mathrm{C} / \mathrm{min}$ & макс./max & 10 \\
\hline Диапазон давлений, Па & мин./min & $10^{-3}$ \\
\cline { 2 - 3 } Pressure range, $\mathrm{Pa}$ & макс./max & $2 \cdot 10^{5}$ \\
\hline $\begin{array}{l}\text { Исследуемые массы, a.e.м. } \\
\text { Investigated masses, a.m.u. }\end{array}$ & мин./min & 2 \\
\cline { 2 - 3 } & макс./max & 100 \\
\hline
\end{tabular}

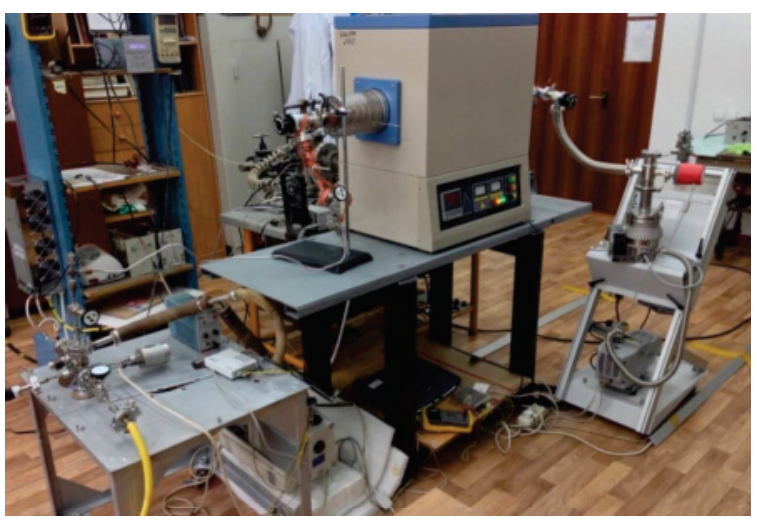

Рис.3. Внешний вид установки для исследования коррозии ма териалов CorrSiC'a

Fig. 3. Appearance of the CorrSiC'a facility for studying materials corrosion

\section{Методика проведения исследований}

Подготовка образца к исследованиям заключалась в его очистке от остатков графитового порошка и углеродных отложений. Для этого образец подвергался ультразвуковой мойке в дважды дистиллированной воде. Далее образец выдерживался при температуре $300{ }^{\circ} \mathrm{C}$ в течении 2 часов в муфельной печи, после проводилось его контрольное взвешивание с помощью аналитических весов Sartorius CPA225D (точность 10 мкг). Затем проводилось исследование состояния его поверхности.

После подготовки к испытаниям образец загружался в центр нагреваемой зоны печи в тигле, устанавливались тепловые экраны, печь герметизировалась торцевыми вакуумными фланцами, и включалась вакуумная откачка. При достижении уровня вакуума 0,1 Па включалась регистрация газового состава (с помощью масс-анализатора) и осуществлялся нагрев образца с выбранной скоростью нагрева (5 или $10{ }^{\circ} \mathrm{C} / \mathrm{мин)}$. Образец нагревался до максимальной температуры $1400{ }^{\circ} \mathrm{C}$ в условиях продолжающейся откачки. Необходимо отметить, что минимальное достигаемое давление 0,1 Па определялось парциальным давлением паров воды, десорбируемых с внутренней поверхности трубы печи. При этом остаточные парциальные давления азота и кислорода (натекание через торцевые фланцы) были на 2-3 порядка ниже. Масс-спектрометр непрерывно регистрировал содержание следующих компонентов (массовых чисел): 2 (водород, $\left.\mathrm{H}_{2}\right), 14-\mathrm{N}(\mathrm{CO}-28), 16-\mathrm{O}\left(\mathrm{CH}_{4}\right)$, 17-OH (компонента воды), 18 (вода, $\mathrm{H}_{2} \mathrm{O}$ ), $28\left(\mathrm{CO}+\mathrm{N}_{2}\right), 32\left(\mathrm{O}_{2}\right), 44\left(\mathrm{CO}_{2}\right)$.

Для создания атмосферы водяного пара при коррозии использовали высокочистую деионизированную дважды дистиллированную воду. Для снижения равновесной концентрации растворенного в воде кислорода ее прогревали в подготовительной камере при температуре $60-70{ }^{\circ} \mathrm{C}$ и давлении 0,2 бар, что, согласно расчетам [28], снижает содержание кислорода в воде до 3-4 мг/кг. Далее при температуре $150-180{ }^{\circ} \mathrm{C}$ и давлении 150-200 кПа вода переводилась в пар, который подавался с помощью импульсного вентиля из накопителя испарителя (объемом $\sim 4$ мл) в камеру печи с образцом (объемом 3,2 л). Давление пара при этом падало до 100 Па. Окончательное значение давления пара в печи измерялось с помощью датчиков давления и регистрировалось компьютером. Поскольку эксперимент проходил при отключенной откачке печи, давление водяного пара в ней не менялось в ходе эксперимента.

Регистрация кинетики изменения химического состава газов начиналась сразу же после напуска водяного пара в камеру с образцом и продолжалась в течение 10-15 минут. После фиксации равновесной интенсивной коррозии образца в камере печи, с помощью выходного клапана системы откачки остатки водяного пара и продукты химических реакций удалялись из печи. Откачка камеры продолжалась до достижения фонового уровня давления $(0,1$ Па), после чего температура печи снижалась до следующего требуемого значения. Аналогично регистрировались коррозионные процессы при $1400,1300,1200,1100,1000,900,800,750{ }^{\circ} \mathrm{C}$. 


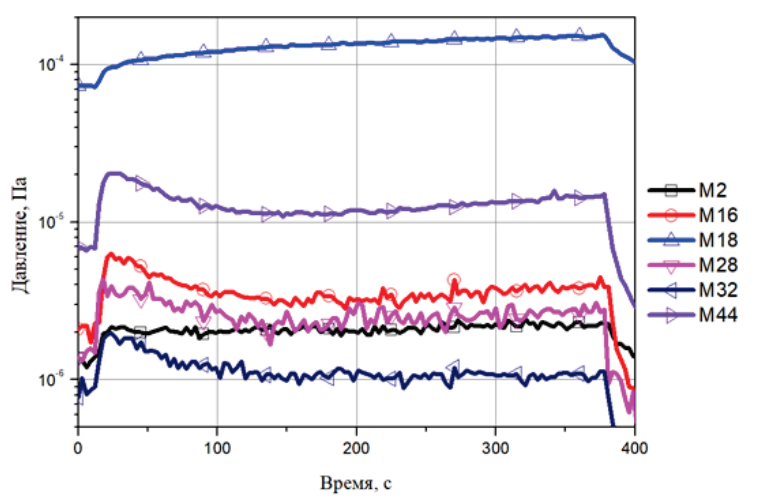

$\mathrm{T}=1400^{\circ} \mathrm{C}$

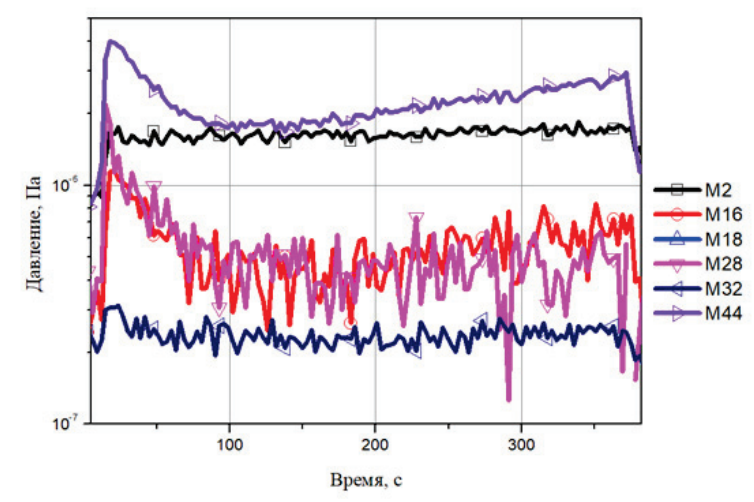

$\mathrm{T}=1200^{\circ} \mathrm{C}$

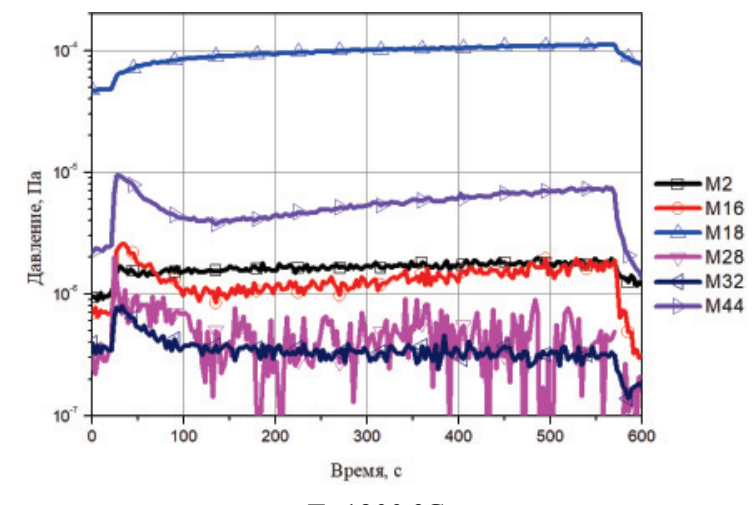

$\mathrm{T}=1300{ }^{\circ} \mathrm{C}$

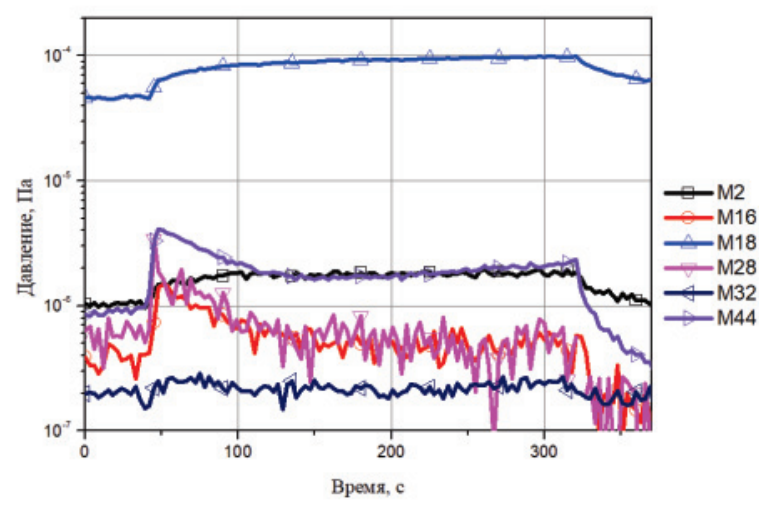

$\mathrm{T}=1100{ }^{\circ} \mathrm{C}$

Pис. 4. Временные зависилости изменения состава газа в камере с образиом графита при подаче паров воды (в легенде указаны массовые числа газов)

Fig. 4. Dependences of gas composition change in the chamber with a graphite sample at water vapor supply (mass numbers of gases are indicated in the legend)

После завершения исследования образца его охлаждали до комнатной температуры, заново взвешивали и проводили послекоррозионные исследования его поверхности и микроструктуры.

Таким образом, в ходе одного ступенчатого снижения температуры образца с 1400 до $700{ }^{\circ} \mathrm{C}$ регистрировались 7-8 различных по интенсивности и длительности коррозионных процессов. Данная методика нам представляется оптимальной с точки зрения определения скорости высокотемпературной коррозии графита с SiC-покрытием в парах воды и/или воздухе.

\section{Результаты экспериментов}

На рис. 4 приведены характерные зависимости изменения химического состава (парциальных давлений) основных газовых составляющих коррозионного процесса графита IG-110 c SiC-покрытием при напусках $\sim 100$ Па паров воды при различных температурах коррозии.

На типичной кривой изменения давления в коррозионной камере с исследуемым образцом $\mathrm{C} / \mathrm{SiC}$ после впрыска туда водяного пара можно выделить несколько характерных участков (рис. 5): 1-2 - напуск водяного пара в корозион- ную камеру; 2-3 - установление равновесного процесса; 3-4 - линейный рост давления (коррозионный процесс); 4 - момент откачки камеры.

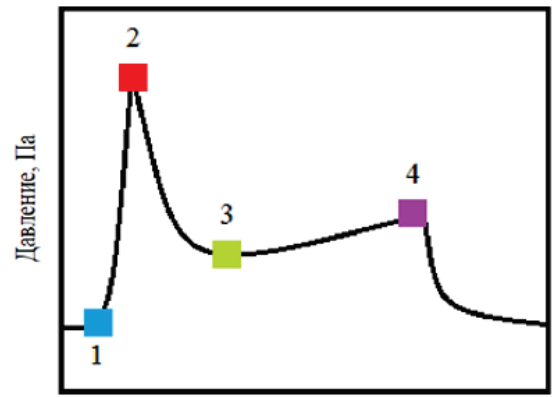

Bремя, c

Pис.5. Характерные участки типичной кривой изменения $\partial а$ вления в зависимости от времени газа в камере с образиом графита при подаче паров воды

Fig. 5. Areas of a typical curve of gas pressure change in a chamber with a graphite sample at water vapor supply

\section{Реакции и механизмы коррозии SiC-покрытий}

Полученные зависимости (рис. 4, 5) определяются целым рядом физико-химических процессов, 


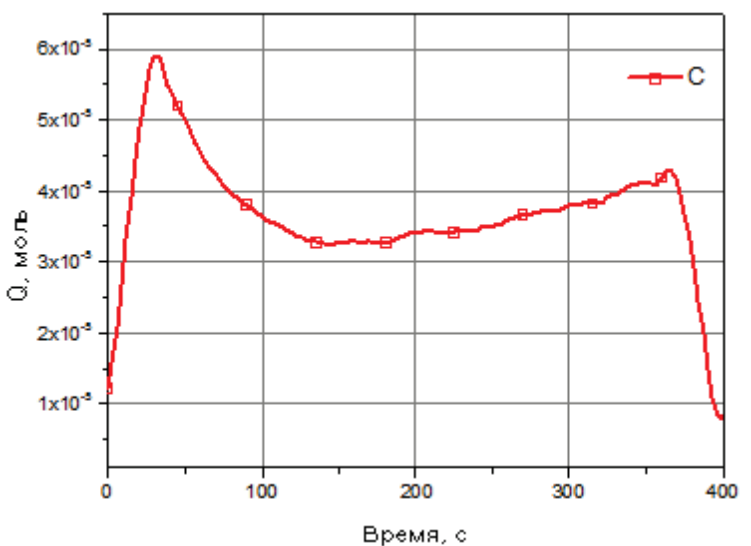

$\mathrm{T}=1400^{\circ} \mathrm{C}$

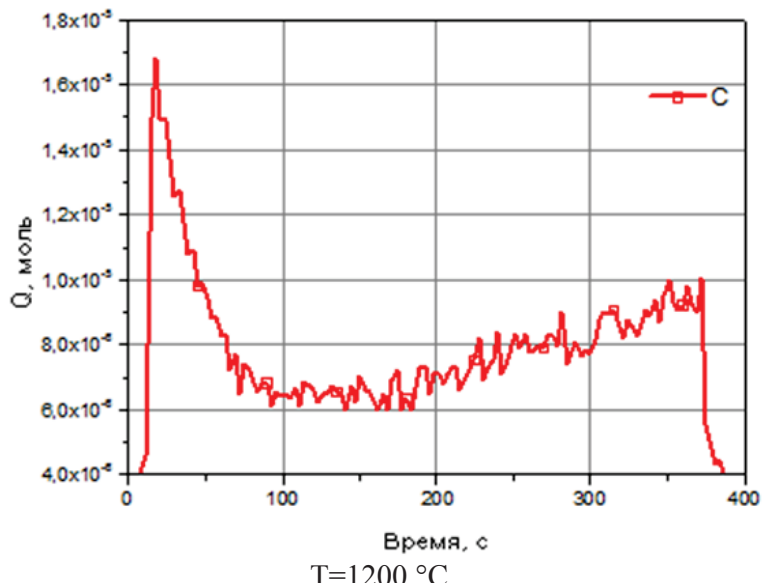

$\mathrm{T}=1200{ }^{\circ} \mathrm{C}$

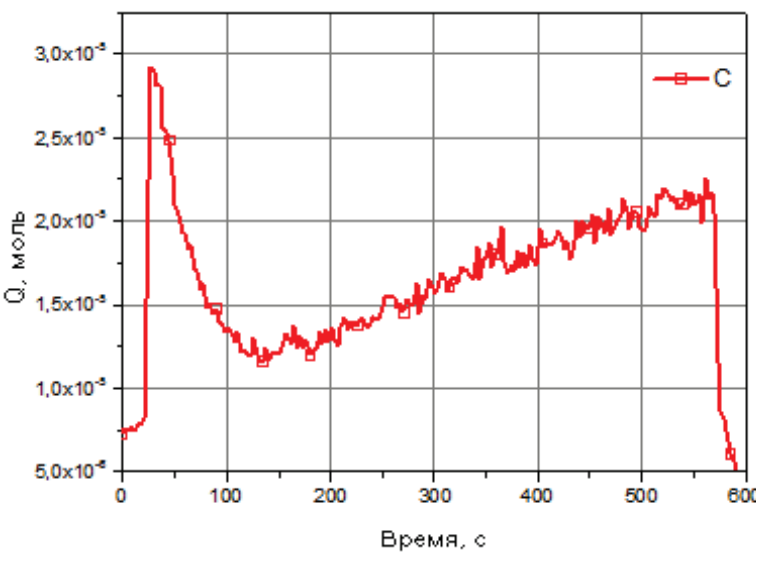

$\mathrm{T}=1300{ }^{\circ} \mathrm{C}$

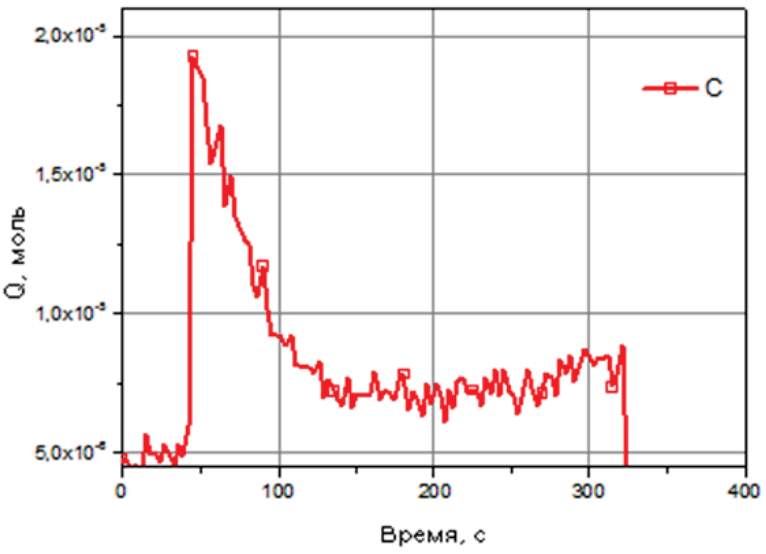

$\mathrm{T}=1100{ }^{\circ} \mathrm{C}$

Puc. 6. Временная зависимость количества атомов углерода в рабочей камере при различных температурах

Fig. 6. Time dependence of number of carbon atoms in the working chamber at different temperatures

таких как: сорбция молекул газов из газовой фазы на поверхность, распад этих молекул на атомы, химические реакции атомов газов на поверхности с атомами поверхности, рекомбинация атомов и их десорбция [15-18, 21-26]. Приведем наиболее важные из вышеперечисленных процессов:

1) активное окисление $\mathrm{SiC}$ (образование газообразного $\mathrm{SiO}$, убыль массы образца):

$\mathrm{SiC}(\mathrm{Tв})+2 \mathrm{H}_{2} \mathrm{O}\left(\right.$ г) $\rightarrow \mathrm{SiO}(г)+2 \mathrm{H}_{2}($ г) $+\mathrm{CO}(г) ;$

2) пассивное окисление $\mathrm{SiC}$ (образование твердого $\mathrm{SiO}_{2}$, прирост массы образца):

$$
\mathrm{SiC}(\mathrm{Tв})+3 \mathrm{H}_{2} \mathrm{O}\left(\text { г) } \rightarrow \mathrm{SiO}_{2}(\mathrm{Tв})+3 \mathrm{H}_{2}(\text { г) }+\mathrm{CO}(\text { г). }\right.
$$

Здесь индексы (тв) и (г) обозначают твердую и газообразную фазы продукта, соответственно.

Важны также реакции взаимодействия газов в коррозионной камере:

А) реакция водорода с СО (образование метана):

$$
3 \mathrm{H}_{2}+\mathrm{CO} \rightarrow \mathrm{CH}_{4}+\mathrm{H}_{2} \mathrm{O}
$$

Б) реакция молекулы воды с СO:

В) распад метана:

$$
\mathrm{H}_{2} \mathrm{O}+\mathrm{CO} \rightarrow \mathrm{CO}_{2}+\mathrm{H}_{2}
$$

$$
\mathrm{CH}_{4} \rightarrow 2 \mathrm{H}_{2}+\mathrm{C} \text {. }
$$

\section{Расчет константы скорости взаимодействия пар-SiC-покрытие}

Как видно из приведенных выше данных, основными продуктами коррозии, доступными для масс-анализа, являются углеродосодержащие газы и водород [15-18, 28-34]. Определить концентрацию газообразного $\mathrm{SiO}$ с помощью квадрупольного масс-анализатора не представляется возможным, т. к. монооксид кремния затвердевает уже при температурах $120-150{ }^{\circ} \mathrm{C}$ и высаживается на холодных элементах системы пробозабора массанализатора, не доходя до ионизатора, поэтому в балансе не учитывается. В этой ситуации основным контролируемым элементом, определяющим коррозию образца, является углерод в составе образующихся углеродсодержащих газов [35]. При

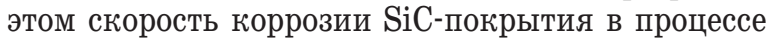
его взаимодействия с парами воды напрямую связана со скоростью увеличения концентрации «газообразного» углерода в коррозионной камере.

Таким образом, используя полученные экспериментальные данные и исходя из перечисленных реакций (1)-(5), можно рассчитать временные за- 
висимости изменения концентрации атомов углерода, убывающего из образца в виде оксидов углерода и метана при различных температурах и определить скорость взаимодействия паров воды с $\mathrm{SiC}$-покрытием.

На рис. 6 приведены кривые изменения концентрации углерода, отделившегося от образца после напуска в коррозионную камеру 100 Па воды, полученные из данных рис. 4. Далее из полученных временных зависимостей для участков 3-4 (рис. 5) определяется скорость наработки углерода как $d Q(t) / d t$. Для этого проводится линеаризация участка 3-4 и определяется наклон прямой.

Затем константа скорости взаимодействия $\mathrm{H}_{2} \mathrm{O}$ c SiC-покрытием определяется как:

$$
k=\frac{d Q}{d t} /\left(P_{\mathrm{H}_{2} \mathrm{O}} \cdot S\right),
$$

где $P_{\mathrm{H}_{2} \mathrm{O}}$ - давление паров воды, напущенных в рабочую камеру; $S$ - площадь поверхности образца. Таким образом, были получены значения константы скорости взаимодействия водяного пара с образцом графита ВТГР для различных температур образца (рис. 7).

Полученная зависимость может быть записана следующим образом:

$$
(T)=106\left(\frac{\text { моль }}{(\mathrm{c} \cdot \Pi \mathrm{\Pi a} \cdot \mathrm{m})}\right) \cdot \exp \left(-\frac{164(\text { кДж/моль })}{R T}\right) .
$$
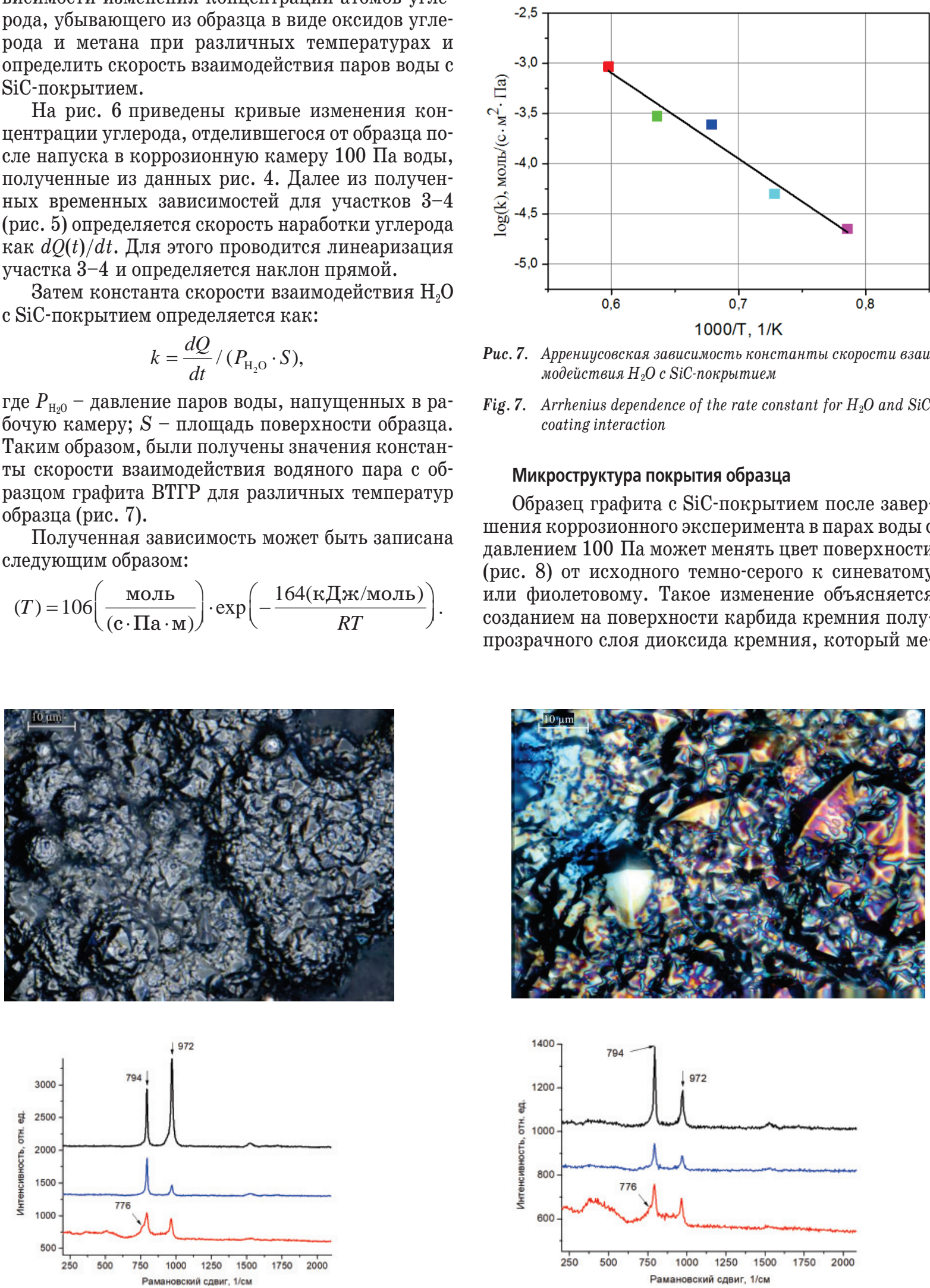

Рис. 7. Аррениусовская зависимость константы скорости взаи модействия $\mathrm{H}_{2} \mathrm{O}$ с SiC-покрытием

Fig. 7. Arrhenius dependence of the rate constant for $\mathrm{H}_{2} \mathrm{O}$ and $\mathrm{SiC}$ coating interaction

\section{Микроструктура покрытия образца}

Образец графита с $\mathrm{SiC}$-покрытием после завершения коррозионного эксперимента в парах воды с давлением 100 Па может менять цвет поверхности (рис. 8) от исходного темно-серого к синеватому или фиолетовому. Такое изменение объясняется созданием на поверхности карбида кремния полупрозрачного слоя диоксида кремния, который ме-
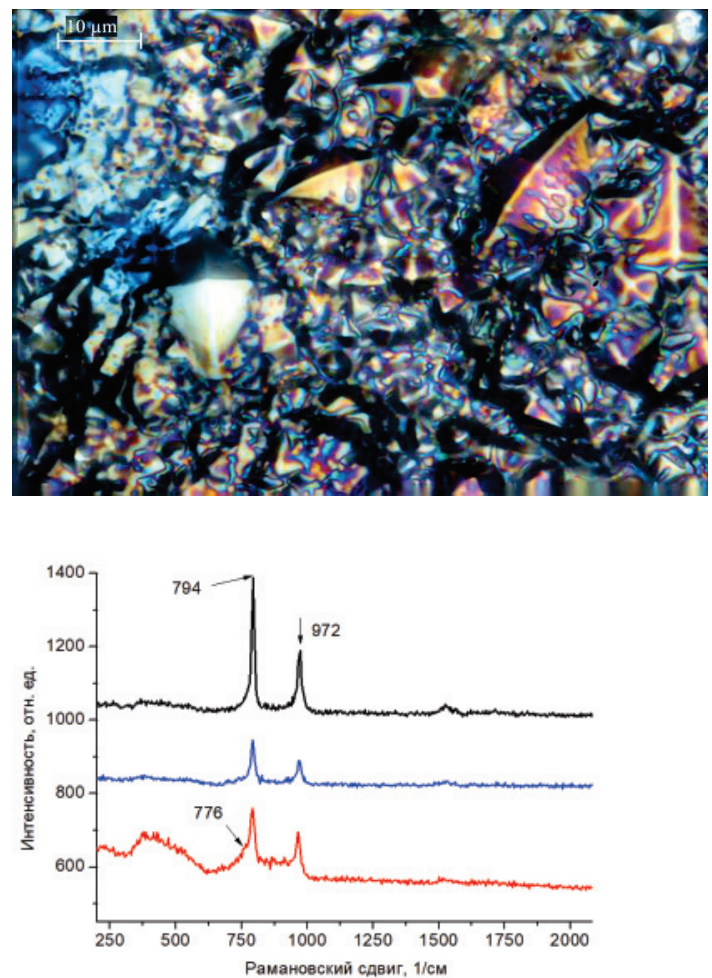

Рис. 8. Результаты микроструктурных исследований поверхности образиа и Рамановские спектры (слева исходный, справа - после коррозии)

Fig. 8. Results of microstructural studies of the sample surface and the Raman spectra (on the left is original, on the right is after corrosion) 
няет оттенок в зависимости от толщины пленки. Согласно литературным данным, красновато-фиолетовый цвет может соответствовать толщине $\mathrm{SiO}_{2}$ пленки 100 мк [20, 31-33, 35].

В образце, помимо существенного изменения цветовых характеристик, наблюдаются и некоторые изменения в структуре. Рамановские пики исходного образца при 794 и $972 \mathrm{~cm}^{-1}$ соответствуют оптическим колебательным модам TO и LO кубической фазы карбида кремния 3C-SiC. B некоторых областях образца на спектрах наблюдается появление плеча в области $776 \mathrm{~cm}^{-1}$, что характерно для ТО мод гексагональных фаз $4 \mathrm{H}-\mathrm{SiC}$ и $6 \mathrm{H}-\mathrm{SiC}$ [36]. После коррозии заметно уменьшается интенсивность пиков 794 и $972 \mathrm{~cm}^{-1}$, а в некоторых областях можно заметить их уширение, что может свидетельствовать о начале процесса аморфизации исходной кристаллической структуры $\mathrm{SiC}$. Bec образца увеличивается примерно на 0,05-0,1\%, в отличие от 1-2 \% убыли веса контрольного образца чистого графита после такого же эксперимента.

Таблица 2. Результаты ЭДС-анализа образиа до и после корро зионных испытаний

Table 2. Results of the EDS analysis of the sample before and after corrosion tests

\begin{tabular}{|c|c|c|}
\hline $\begin{array}{c}\text { Элемент } \\
\text { Element }\end{array}$ & $\begin{array}{c}\text { До испытания, мас. \% } \\
\text { Before tests, wt. \% }\end{array}$ & $\begin{array}{c}\text { После испытания, мac. \% } \\
\text { After tests, wt. \% }\end{array}$ \\
\hline $\mathrm{Si}$ & 60,47 & 60,10 \\
\hline 0 & 0,30 & 23,17 \\
\hline $\mathrm{C}$ & 39,23 & 16,73 \\
\hline Bcero/Total & 100 & 100 \\
\hline
\end{tabular}

Результаты ЭДС-анализа (энергодисперсионная спектроскопия) образца после пассивной коррозии свидетельствуют о присутствии на поверх-

\section{СПИСОК ЛИТЕРАТУРЫ}

1. Tazhibaeva I.L., Kulsartov T.V., Kenzhin E.A. Material Science Activities for Fusion Reactors in Kazakhstan // Journal of the Nuclear Materials. - 2009. - V. 386-388. - P. 15-18.

2. Tritium migration in the materials proposed for fusion reactors: $\mathrm{Li}_{2} \mathrm{TiO}_{3}$ and beryllium / T.V. Kulsartov, Yu.N. Gordienko, I.L. Tazhibayeva, E.A. Kenzhin, N.I. Barsukov, A.O. Sadvakasova, A.V. Kulsartova, Zh.A. Zaurbekova // Journal of the Nuclear Materials. - 2013. - V. 442. - P. 740-745.

3. Research of Reactor Radiation Influence upon Processes of Hydrogen Isotopes Interaction with Materials of the Fusion Facility / A.0. Sadvakassova, T.V. Kulsartov, I.L. Tazhibaeva, E.A. Kenzhin // Fusion Science and Technology. - 2011. V. 60 (1T). - P. 9-15.

4. Status of design and experimental activity on module of lithium divertor of KTM tokamak / I. Lyublinski, A. Vertkov, M. Zharkov, V. Semenov, S. Mirnov, V. Lazarev, I. Tazhibayeva, G. Shapovalov, V. Dyachenko, A. Azizov, G. Mazzitelli, P. Agostini // Fusion Engineering and Design. - 2013. - V. 88. - Iss. 9-10. P.1862-1865.

5. Детритизация облученного бериллия различных марок тдс методом / Т.В. Кульсартов, И.Л. Тажибаева, Ю.Н. Гордиенко, Ж.А. Заурбекова, Е.Т. Коянбаев, И.М. Кукушкин, Е.А. Кенжин, А.О. Муканова, Д.С. Дюсамбаев, А.А. Шаймерденов, ности значительных концентраций кислорода (табл. 2), что подтверждает наличие тонкого слоя (около 100 мк) аморфного стекла $\mathrm{SiO}_{2}$.

\section{Заключение}

Проведены эксперименты по высокотемпера-

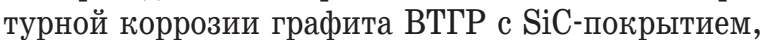
и получены температурные зависимости изменения газового выхода коррозии для температур от 750 до $1400{ }^{\circ} \mathrm{C}$ при впрыске 100 Па водяного пара.

Установлено, что реакция $\mathrm{SiC}$-покрытия графита с водяным паром при давлении 100 Па и выше происходит по пассивному (образующему дополнительный защитный слой диоксида кремния) механизму. Коррозия $\mathrm{SiC}$ в парах воды происходит при температурах $1100-1400{ }^{\circ} \mathrm{C}$. В диапазоне температур 750-900 ${ }^{\circ} \mathrm{C}$ коррозия почти не регистрируется масс-анализатором.

Разработана модель для аналитического описания результатов экспериментов и рассчитаны скорости реакции окисления карбида кремния графита с парами воды при $1000-1400{ }^{\circ} \mathrm{C}$.

Анализ изменения микроструктуры образцов после коррозионного эксперимента свидетельствует об образовании на поверхности карбида кремния защитного слоя $\mathrm{SiO}_{2}$, характерного для механизма пассивного окисления $\mathrm{SiC}$.

Результаты исследований позволяют заключить, что графит с $\mathrm{SiC}-$-покрытием обладает существенно более низкой скоростью коррозии (например, по сравнению с графитом, скорость коррозии отличается более чем в 1000 раз) и может быть применен для защиты топлива и графитовых узлов ВТГР.

Работа выполнена при поддержке Министерства образования и науки Республики Казахстан в рамках проекma № AP05132169.

Л.В. Чекушина // ВАНТ. Сер. Термоядерный синтез - 2014. T. 37. - Вып. 2. - С. 27-37.

6. Properties of tritium/helium release from hot isostatic pressed beryllium of various trademarks / L. Chekushina, D. Dyussambaev, A. Shaimerdenov, K. Tsuchiya, T. Takeuchi, H. Kawamura, T. Kulsartov // Journal of the Nuclear Materials. - 2014. V. $452 .-$ P. $41-45$

7. Investigation of hydrogen permeability through copper alloy $\mathrm{CuCr} 1 \mathrm{Zr} 0.1$ and duplex structure $\mathrm{Be}-\mathrm{Cu} / \mathrm{T} . \mathrm{V}$. Kulsartov, Y.V. Chikhray, I.L. Tazhibaeva, V.P. Shestakov // Fusion Technology. - 1998. - V. 34. - Iss. 3. - Part 2. - P. 919-923.

8. Study of $\mathrm{Li}_{2} \mathrm{TiO}_{3}+5 \mathrm{~mol} \% \mathrm{TiO}_{2}$ lithium ceramics after long-term neutron irradiation / Y. Chikhray, V. Shestakov, 0. Maksimkin, L. Turubarova, I. Osipov, T. Kulsartov, A. Kuykabayeba, I. Tazhibayeva, H. Kawamura, K. Tsuchiya // Journal of the Nuclear Materials. - 2009. - V. 386-388. - P. 286-289.

9. Mechanism of Tritium accumulation and release from $\mathrm{Li}_{2} \mathrm{TiO}_{3}$ ceramics during long-term irradiation at WWR-K Reactor / I.L. Tazhibaeva, T.V. Kulsartov, I. Beckman, V.P. Shestakov, E.A. Kenzhin, E.V. Chikhray, H. Kawamura, A. Kiykabaeva, K. Tsuchiya // Journal of the Nuclear Materials. - 2011. V. 411. - P. 121-126.

10. In-pile assemblies for investigation of tritium release from $\mathrm{Li}_{2} \mathrm{TiO}_{3}$ lithium ceramic / V. Shestakov, I. Tazhibayeva, H. Kawamura, E.A. Kenzhin, E.V. Chikhray, T.V. Kulsartov, A. Kolbaen- 
kov, F. Arinkin, Sh. Kh. Gizatulin, P. Chakrov // Fusion Science and Technology. - 2005. - V. 47. - Iss. 4. - P. 1084-1088.

11. Investigation of the surface element composition influence on hydrogen permeability through vanadium alloy $\mathrm{VCr}_{4} \mathrm{Ti}_{4}$ I.V. Shkolnik, T.V. Kulsartov, I.L. Tazhibaeva, V.P. Shestakov // Fusion Technology. - 1998. - V. 34. - Iss. 3. - Part 2. P. $868-871$.

12. Mullite based oxidation protection for $\mathrm{SiC} / \mathrm{C}$ composites in air at temperature up to $1900 \mathrm{~K} / \mathrm{H}$. Fritze, J. Jojie, T. Witke, C. Ruscher, S. Weber, S. Scherrer et al. // J. Eur. Ceram. Soc. 1998. - V. 18. - № 16. - P. 2351-2364.

13. Huang J.F., Zeng X.R., Li H.J. Mullite- $\mathrm{Al}_{2} \mathrm{O}_{3}-\mathrm{SiC}$ oxidation protective coating for $\mathrm{C} / \mathrm{C}$ composites // Carbon. -2003 . - V. 41. № 14. - P. 2825-2829.

14. Создание труб-оболочек твәлов из композиционных материалов на основе карбида кремния / Ф. Макаров, А. Пономаренко, Р. Захаров, И. Дзюбинский, С. Иванов, А. Глебов, М. Лебедев // Наноиндустрия. - 2017. - Т. 73. - № 3. - С. 60-67.

15. Thermodynamic Analysis of Behavior of HTGR Fuel and Fission Products under Accidental Air or Water Ingress Conditions / K. Minato, K. Fukuda // Proc. IAEA Technical Committee Mtg. on Response of Fuel, Fuel Elements and Gas-cooled Reactor Cores under Accidental Air or Water Ingress Conditions, IAEA-TECD0C-784. - Beijing, China, 1995. - P. 86-91.

16. Действие облучения на графит ядерных реакторов / В.В. Гончаров, Н.С. Бурдаков, Ю.С. Виргильев и др. - М.: Атомиздат, 1978. $-272 \mathrm{c.}$

17. Odeychuk M.P. Graphites and CCCM for nuclear reactor plants: Ukraine's experience // Proc. of International Conference on Ceramic Processing. - Mumbai, India, 2004. - P. 103-123.

18. Ferng Y.M., Chi C.W. CFD investigating the air ingress accident for a HTGR simulation of graphite corrosion oxidation // Nuclear Engineering and Design. - 2012. - V. 248. - P. 55-65.

19. Chikhray Y. et al. Corrosion test of HTGR graphite with SiC-coating // ANS Proceedings. International Topical Meeting on High Temperature Reactor Technology (HTR 2016). - Las Vegas, NV, November 6-10, 2016. - V. 1. - P. 572-577.

20. International Business Machines Corporation. Color Chart for Thermally Grown $\mathrm{SiO}_{2}$. URL: http://www.htelabs.com/appnotes/sio2_color_chart_thermal_silicon_dioxide.htm (дата обращения $\overline{25} .03 . \overline{2} 019$ ).

21. Wang P., Yu X., Yu S. Progress of the study on corrosion behavior of graphite in HTGRs // Proc. of the $18^{\text {th }}$ International Conference on Nuclear Engineering, ICONE18. - Xi'an, China, May 17-21, 2010. - V. 5. - P. 1-7.

22. Beyond the classical kinetic model for chronic graphite oxidation by moisture in high temperature gas-cooled reactors / C.I. Contescu, R.W. Mee, Y. Lee, J.D. Arregui-Mena, N.C. Gallego, T.D. Burchell, J.J. Kane, W.E. Windes // Carbon. - 2018. V. 127. - P. 158-169.

23. Effective gaseous diffusion coefficients of select ultra-fine, super-fine and medium grain nuclear graphite / J.J. Kane, A.C. Matthews, C.J. Orme, C.I. Contescu, W.D. Swank, W.E. Windes // Carbon. - 2018. - V. 136. - P. 369-379.

24. Characterizing thermal-oxidation behaviors of nuclear graphite by combining $\mathrm{O}_{2}$ supply and micro surface area of graphite Y. Zhou, Y. Dong, H. Yin, Z. Li, R. Yan, D. Li, Z. Zhang // Scien- tific Reports. - 2018. - V. 8:13400. DOI: 10.1038/s41598-018-31493-4.

25. Luo X., Jean-Charles R. Research of Oxidation Properties of Graphite Used in HTR-10. URL: https://inis.iaea.org/collection/NCLCollectionStore/_Public/43/024/43024028.pdf (дата обращения 25.03.2019).

26. Thermal analysis of the oxidation of natural graphite-effect of particle size / W. Jiang, G. Nadeau, K. Zaghib, K. Kinoshita // Thermochimica Acta. - 2000. - V. 351. - № 1-2 - P. 85-93.

27. Excellent Feature of Japanese HTGR Technologies / T. Nishihara, X. Yan, Y. Tachibana, T. Shibata, H. Ohashi, S. Kubo, Y. Inaba, S. Nakagawa, M. Goto, S. Ueta, N. Hirota, Y. Inagaki, K. Iigaki, S. Hamamoto, K. Kunitomi // JAEA-Technology 2018-004. - 2018. Japan Atomic Energy Agency. DOI: 10.11484/jaea-technology-2018-004.

28. Иванов М. Термическая деаэрация воды // Аква-Терм. 2008. - T. 41. - № 1. - C. 15-17.

29. Campbell P.A., Mitchell R.E. The Impact of the Distributions of Surface 0xides and their Migration on Characterization of the Heterogeneous Carbon-0xygen Reaction // Combustion and Flame. - 2008. - V. 154. - P. 47-66.

30. 0 строении систем $\mathrm{SiC}-\mathrm{B}_{4} \mathrm{C}-\mathrm{Me}^{\mathrm{d}} \mathrm{B}_{2}$ и перспективах создания композиционных керамических материалов на их основе / С.С. Орданьян, Д.Д. Несмелов, Д.П. Данилович, Ю.П. Удалов // Известия вузов. Порошковая металлургия и функциональные покрытия. - 2016. - Т. 4. - С. 41-50.

31. Ларионов Н.В. Покрытия из карбида кремния, полученные пиролизом монометилсилана, на реакционно-связанном карбиде кремния // Информационно-технологический вестник. 2018. - № 1. - С. 127-136.

32. Влияние воздействия высокоскоростного потока азота на структуру и химический состав высокотемпературного покрытия на композиционном SiC-материале / Б.Е. Жестков, М.Л. Ваганова, Ю.Е. Лебедева, 0.Ю. Сорокин, П.Н. Медведев // Теплофизика высоких температур. - 2018. - Т. 56. № 3. - C. 395-398.

33. Шикунов С.Л., Курлов В.Н. Получение композиционных материалов на основе карбида кремния силицированием углеродных матриц // Журнал технической физики. - 2017. - Т. 87. № 12. - C. 1871-1878.

34. Исследование износостойкости композиционного керамического материала системы TiC-SiC, полученного плазменно-искровым спеканием / М.Н. Каченюк, О.В. Сомов, Н.Б. Асташина, К.Э. Андраковская, Н.В. Морозова // Электронная обработка материалов. - 2016. - Т. 52. - № 6. - С. 19-24.

35. Investigation of the 0xidative Resistance of High-Temperature Coating on a $\mathrm{SiC}$ Material under Exposure to High-Enthalpy Flow / E.N. Kablov, B.E. Zhestkov, D.V. Grashchenkov, 0.Yu. Sorokin, Yu.E. Lebedeva, M.L. Vaganova // High Temperature. -2017 . - V. 55. - № 6. - P. 873-879.

36. Optical spectroscopy study of damage induced in $4 \mathrm{H}-\mathrm{SiC}$ by swift heavy ion irradiation / S. Sorieul, X. Kerbiriou, J-M. Costantini, L. Gosmain, G. Calas, C. Trautmann // J. Phys.: Condens. Matter. - 2012. - V. 24. - Article № 125801 (7 p.).

Поступила 03.04.2019 2. 


\section{Информация об авторах}

Аскербеков C.K., младший научный сотрудник лаборатории конструкционных материалов для ядерно-энергетических установок НИИ «Экспериментальной и теоретической физики» Казахского Национального Университета им. аль-Фараби.

Чихрай E.B., кандидат технических наук, ведущий научный сотрудник лаборатории конструкционных материалов для ядерно-энергетических установок НИИ «Экспериментальной и теоретической физики» Казахского Национального Университета им. аль-Фараби.

Понкратов Ю.В., научный сотрудник лаборатории внутриканальных испытаний Института атомной энергии Национального ядерного центра Республики Казахстан.

Никитенков Н.H., доктор физико-математических наук, профессор отделения экспериментальной физики Инженерной школы ядерных технологий национального исследовательского Томского политехнического университета. 
UDC $620.19,621.039 .586,621.039 .524 .2 .034 .3$

\title{
RESEARCH OF HIGH-TEMPERATURE CORROSION OF SIC-COATING ON GRAPHITE
}

\author{
Saulet K. Askerbekov',
}

askerbekov@physics.kz

\section{Evgeniy V. Chikhray', \\ chikhray@physics.kz}

\section{Yuriy V. Ponkratov ${ }^{2}$, ponkratov@nnc.kz}

\author{
Nikolay N. Nikitenkov ${ }^{3}$, \\ nikitenkov@tpu.ru \\ ${ }^{1}$ Kazakh National University named after Al-Farabi, \\ 71, Al-Farabi Avenue, Almaty, 050040, Kazakhstan. \\ 2 National Nuclear Center of the Republic of Kazakhstan, \\ 10, Krasnoarmeyskaya street, Kurchatov, 071100, Kazakhstan. \\ ${ }^{3}$ National Research Tomsk Polytechnic University, \\ 30, Lenin avenue, Tomsk, 634050, Russia.
}

The relevance of this work is caused by possible prospect of creating a Kazakhstani high-temperature gas-cooled reactor, in which a number of new materials science solutions will be applied regarding the graphite matrix and fuel cells. Silicon carbide (SiC) coatings will be used on reactor fuel cells, in which graphite is the main constituent material.

The aim of the research is to evaluate the corrosion rate of reactor graphite with SiC-coating in the temperature range from 750 to $1400{ }^{\circ} \mathrm{C}$ at the initial pressure of water vapor in the chamber $100 \mathrm{~Pa}$.

Object: isotropic fine-grained graphite of IG-110 brand with a protective SiC-coating thickness of 200 microns.

Methods: programmable thermal desorption of samples in the presence of water vapor, mass spectrometric recording of the gas composition in the working chamber, microstructural studies, Raman spectroscopy, energy-dispersed X-ray spectroscopy.

Results. It is shown that at pressures of water vapor of $100 \mathrm{~Pa}$, the SiC-coating interacts with steam through a passive (forming an additional protective layer of amorphous silicon dioxide) mechanism. Corrosion of SiC in water vapor at temperatures of $1100-1400{ }^{\circ} \mathrm{C} \mathrm{oC}-$ curs by orders of magnitude more intense than at $750-900^{\circ} \mathrm{C}$. A model for analytical description of the experimental results was developed and the parameters of the rate constant for water vapor interaction with a SiC-coating were calculated for sample temperatures of $1400,1300,1200,1100$, and $1000^{\circ} \mathrm{C}$. Microstructural studies of the samples before and after corrosion tests were carried out, which showed that a protective $\mathrm{SiO}_{2}$ film is formed on the surface with a SiC carbide coating during passive corrosion. The research results allow us to conclude that the prototype of a fuel with a SiC-coating has improved anti-corrosion properties and can be successfully used to reduce carbon chemical activity in fuel cells and fuel assemblies of high-temperature gas-cooled reactor without deteriorating their mechanical and temperature properties.

\section{Key words:}

Reactor graphite, SiC-coating, HTGR, corrosion, water vapor.

This work was supported by the Ministry of Education and Science of the Republic of Kazakhstan in the framework of the project No. AP05132169.

\section{REFERENCES}

1. Tazhibaeva I.L., Kulsartov T.V., Kenzhin E.A. Material Science Activities for Fusion Reactors in Kazakhstan. Journal of the Nuclear Materials, 2009, vol. 386-388, pp. 15-18.

2. Kulsartov T.V., Gordienko Yu.N., Tazhibayeva I.L., Kenzhin E.A., Barsukov N.I., Sadvakasova A.0., Kulsartova A.V., Zaurbekova Zh.A. Tritium migration in the materials proposed for fusion reactors: $\mathrm{Li}_{2} \mathrm{TiO}_{3}$ and beryllium. Journal of the Nuclear Materials, 2013, vol. 442, pp. 740-745.

3. Sadvakassova A.O., Kulsartov T.V., Tazhibaeva I.L., Kenzhin E.A. Research of Reactor Radiation Influence upon Processes of Hydrogen Isotopes Interaction with Materials of the Fusion Facility. Fusion Science and Technology, 2011, vol. 60, no. 1, pp. 9-15.

4. Lyublinski I., Vertkov A., Zharkov M., Semenov V., Mirnov S., Lazarev V., Tazhibayeva I., Shapovalov G., Dyachenko V., Azizov A., Mazzitelli G., Agostini P. Status of design and experimental activi- ty on module of lithium divertor of KTM tokamak. Fusion Engineering and Design, 2013, vol. 88, no. 9-10, pp. 1862-1865.

5. Kulsartov T.V., Tazhibayeva I.L., Gordiyenko Yu.N., Zaurbekova Zh.A., Koyanbayev E.T., Kukushkin I.M., Kenzhin E.A., Mukanova A.O., Dyusambayev D.S., Shaymerdenov A.A., Chekushina L.V. Detritization of irradiated beryllium of various grades using tds method. PAS\&T/TF, 2014, vol. 37, no. 2, pp. 27-37. In Rus.

6. Chekushina L., Dyussambaev D., Shaimerdenov A., Tsuchiya K., Takeuchi T., Kawamura H., Kulsartov T. Properties of tritium/helium release from hot isostatic pressed beryllium of various trademarks. Journal of the Nuclear Materials, 2014, vol. 452, pp. 41-45.

7. Kulsartov T.V., Chikhray Y.V., Tazhibaeva, I.L., Shestakov V.P. Investigation of hydrogen permeability through copper alloy CuCr1Zr0.1 and duplex structure Be-Cu. Fusion Technology, 1998, vol. 34, no. 3, P. 2, pp. 919-923.

8. Chikhray Y., Shestakov V., Maksimkin 0., Turubarova L., Osipov I., Kulsartov T., Kuykabayeba A., Tazhibayeva I., Kawamura H., 
Tsuchiya K. Study of $\mathrm{Li}_{2} \mathrm{TiO}_{3}+5 \mathrm{~mol} \% \mathrm{TiO}_{2}$ lithium ceramics af ter long-term neutron irradiation. Journal of the Nuclear Materials, 2009, vol. 386-388, pp. 286-289.

9. Tazhibaeva I.L., Kulsartov T.V., Beckman I., Shestakov V.P., Kenzhin E.A., Chikhray E.V., Kawamura H., Kiykabaeva A., Tsuchiya K.. Mechanism of Tritium accumulation and release from $\mathrm{Li}_{2} \mathrm{TiO}_{3}$ ceramics during long-term irradiation at WWR-K Reactor. Journal of the Nuclear Materials, 2011, vol. 411, pp. 121-126.

10. Shestakov V., Tazhibayeva I., Kawamura H., Kenzhin E.A., Chikhray E.V., Kulsartov T.V., Kolbaenkov A., Arinkin F., Gizatulin Sh.Kh., Chakrov P. In-pile assemblies for investigation of tritium release from Li2Ti03 lithium ceramic. Fusion Science and Technology, 2005, vol. 47, no. 4, pp. 1084-1088.

11. Shkolnik I.V., Kulsartov T.V., Tazhibaeva I.L., Shestakov V.P. Investigation of the surface element composition influence on hydrogen permeability through vanadium alloy $\mathrm{VCr}_{4} \mathrm{Ti}_{4}$. Fusion Technology, 1998, vol. 34, no. 3, part 2, pp. 868-871.

12. Fritze H., Jojie J., Witke T., Ruscher C., Weber S., Scherrer S. Mullite based oxidation protection for $\mathrm{SiC} / \mathrm{C}$ composites in air at temperature up to 1900 K. J. Eur. Ceram. Soc., 1998, vol. 18, no. 16 , pp. $2351-2364$.

13. Huang J.F., Zeng X.R., Li H.J. Mullite- $\mathrm{Al}_{2} \mathrm{O}_{3}$-SiC oxidation protective coating for $\mathrm{C} / \mathrm{C}$ composites. Carbon, 2003, vol. 41, no. 14, pp. 2825-2829.

14. Makarov F., Ponomarenko A., Zakharov R., Dzyubinskiy I., Ivanov S., Glebov A., Lebedev M. Creating shells of fuel rods made of composite materials based on silicon carbide. Nanoindustry, 2017, vol. 73, no. 3, pp. 60-67. In Rus.

15. Minato K., Fukuda K.. Thermodynamic Analysis of Behavior of HTGR Fuel and Fission Products under Accidental Air or Water Ingress Conditions. Proc. IAEA Technical Committee Mtg. on Response of Fuel, Fuel Elements and Gas-cooled Reactor Cores under Accidental Air or Water Ingress Conditions, IAEA-TECDOC-784. Beijing, China, 1995, pp. 86-91.

16. Goncharov V.V., Burdakov N.S., Virgilyev Yu.S. Deystvie oblucheniya na grafit yadernykh reaktorov [Irradiation effect on graphite of nuclear reactors]. Moscow, Atomizdat Publ., 1978. 272 p.

17. Odeychuk M.P. Graphites and CCCM for nuclear reactor plants: Ukraine's experience. Proc. of International Conference on Ceramic Processing. Mumbai, India, 2004. pp. 103-123.

18. Ferng Y.M., Chi C.W. CFD investigating the air ingress accident for a HTGR simulation of graphite corrosion oxidation. Nuclear Engineering and Design, 2012, vol. 248, pp. 55-65.

19. Chikhray Y. Corrosion test of HTGR graphite with $\mathrm{SiC}$ coating. ANS Proceedings, International Topical Meeting on High Temperature Reactor Technology (HTR 2016). Las Vegas, NV, November $6-10,2016$. Vol. 1, pp. 572-577.

20. International Business Machines Corporation. Color Chart for Thermally Grown $\mathrm{SiO}_{2}$. Available at: http://www.htelabs.com/appnotes/sio2_color_chart_thermal_silicon_dioxide.htm (accessed 25 March 2019).

21. Wang P., Yu X., Yu S. Progress of the study on corrosion behavior of graphite in HTGRs. Proc. of the $18^{\text {th }}$ International Conference on Nuclear Engineering, ICONE18. Xi'an, China, May 17-21, 2010. Vol. 5, pp. 1-7.

22. Contescu C.I., Mee R.W., Lee Y., Arregui-Mena J.D., Gallego N.C., Burchell T.D., Kane J.J., Windes W.E. Beyond the classical kinetic model for chronic graphite oxidation by moisture in high temperature gas-cooled reactors. Carbon, 2018, vol. 127, pp. 158-169.
23. Kane J.J., Matthews A.C., Orme C.J., Contescu C.I., Swank W.D., Windes W.E. Effective gaseous diffusion coefficients of select ultra-fine, super-fine and medium grain nuclear graphite. Carbon, 2018, vol. 136, pp. 369-379.

24. Zhou Y., Dong Y., Yin H., Li Z., Yan R., Li D., Zhang Z. Characterizing thermal-oxidation behaviors of nuclear graphite by combining $\mathrm{O}_{2}$ supply and micro surface area of graphite. Scientific Reports, 2018, vol. 8:13400. DOI: 10.1038/s41598-018-31493-4.

25. Luo X., Jean-Charles R. Research of Oxidation Properties of Graphite Used in HTR-10. https://inis.iaea.org/collection/NCLCollectionStore/_Public/43/024/43024028.pdf (accessed 25 March 2019).

26. Jiang W., Nadeau G., Zaghib K., Kinoshita K. Thermal analysis of the oxidation of natural graphite - effect of particle size. Thermochimica Acta, 2000, vol. 351, no. 1-2, pp. 85-93.

27. Nishihara T., Yan X., Tachibana Y., Shibata T., Ohashi H., Kubo S., Inaba Y., Nakagawa S., Goto M., Ueta S., Hirota N., Inagaki Y., Iigaki K., Hamamoto S., Kunitomi K. Excellent Feature of Japanese HTGR Technologies. JAEA-Technology 2018-004. 2018, Japan Atomic Energy Agency. DOI: 10.11484/jaea-technology-2018-004.

28. Ivanov V. Thermal water deaeration. Aqua-Therm, 2008, vol. 41, no. 1, pp. 15-17. In Rus.

29. Campbell P.A., Mitchell R.E. The Impact of the Distributions of Surface 0xides and their Migration on Characterization of the Heterogeneous Carbon-0xygen Reaction. Combustion and Flame, 2008, vol. 154, pp. 47-66.

30. Ordanyan S.S., Nesmelov D.D., Danilovich D.P., Udalov Yu.P. On constructing the $\mathrm{SiC}-\mathrm{B} 4 \mathrm{C}-\mathrm{MedB} 2$ systems and the prospects for creating composite ceramic materials based on them. News of universities. Powder Metallurgy and Functional Coatings, 2016, vol. 4, pp. 41-50. In Rus.

31. Larionov N.V. Silicon carbide coatings obtained by pyrolysis of monomethylsilane on reaction-bonded silicon carbide. Information and Technological Journal, 2018, no. 1, pp. 127-136. In Rus.

32. Zhestkov B.E., Vaganova M.L., Lebedeva Yu.E., Sorokin O.Yu., Medvedev P.N. The effect of high-speed nitrogen flow on the structure and chemical composition of high-temperature coating on composite $\mathrm{SiC}$-material. Thermal physics of high temperatures, 2018, vol. 56, no. 3, pp. 395-398. In Rus.

33. Shikunov S.L., Kurlov V.N. Production of composite materials based on silicon carbide by siliconizing carbon matrices. Journal of Technical Physics, 2017, vol. 87, no. 12, pp. 1871-1878. In Rus.

34. Kachenyuk M.N., Somov 0.V., Astashina N.B., Andrakovskaya K.E., Morozova N.V. Investigation of wear resistance of composite ceramic material of the TiC-SiC system, obtained by plasma-spark sintering. Electronic material processing, 2016, vol. 52, no. 6, pp. 19-24. In Rus.

35. Kablov E.N., Zhestkov B.E., Grashchenkov D.V., Sorokin 0.Yu., Lebedeva Yu.E., Vaganova M.L. Investigation of the Oxidative Resistance of High-Temperature Coating on a SiC Material under Exposure to High-Enthalpy Flow. High Temperature, 2017, vol. 55 , no. 6 , pp. 873-879.

36. Sorieul S., Kerbiriou X., Costantini J-M., Gosmain L., Calas G., Trautmann C. Optical spectroscopy study of damage induced in $4 \mathrm{H}-\mathrm{SiC}$ by swift heavy ion irradiation. J. Phys.: Condens. Matter., 2012, vol. 24, Article no. 125801 (7 p).

Received: 3 April 2019.

\section{Information about the authors}

Saulet K. Askerbekov, junior researcher, Kazakh National University named after Al-Farabi.

Evgeniy V. Chikhray, Cand. Sc., leading researcher, Kazakh National University named after Al-Farabi.

Yuriy V. Ponkratov, researcher, National Nuclear Center of the Republic of Kazakhstan.

Nikolay N. Nikitenkov, Dr. Sc., professor, National Research Tomsk Polytechnic University. 